\title{
A randomized controlled trial of hospital versus home based therapy with oral amoxicillin for severe pneumonia in children aged 3 - 59 months: The IndiaCLEN Severe Pneumonia Oral Therapy (ISPOT) Study
}

Archana B. Patel ${ }^{1}$, Akash Bang ${ }^{2 *}$, Meenu Singh ${ }^{3}$, Leena Dhande ${ }^{1}$, Luke Ravi Chelliah ${ }^{4}$, Ashraf Malik ${ }^{5}$, Sandhya Khadse ${ }^{6}$ and ISPOT Study Group

\begin{abstract}
Background: Pneumonia is the leading cause of child mortality under five years of age worldwide. For pneumonia with chest indrawing in children aged 3-59 months, injectable penicillin and hospitalization was the recommended treatment. This increased the health care cost and exposure to nosocomial infections. We compared the clinical and cost outcomes of a seven day treatment with oral amoxicillin with the first $48 \mathrm{~h}$ of treatment given in the hospital (hospital group) or at home (home group).

Methods: We conducted an open-label, multi-center, two-arm randomized clinical trial at six tertiary hospitals in India. Children aged 3 to 59 months with chest indrawing pneumonia were randomized to home or hospital group. Clinical outcomes, treatment adherence, and patient safety were monitored through home visits on day 3, 5, 8 , and 14 with an additional visit for the home group at $24 \mathrm{~h}$. Clinical outcomes included treatment failure rates up to 7 days (primary outcome) and between 8-14 days (secondary outcome) using the intention to treat and per protocol analyses. Cost outcomes included direct medical, direct non-medical and indirect costs for a random $17 \%$ subsample using the micro-costing technique.

Results: 1118 children were enrolled and randomized to home $(n=554)$ or hospital group $(n=564)$. Both groups had similar baseline characteristics. Overall treatment failure rate was $11.5 \%$ (per protocol analysis). The hospital group was significantly more likely to fail treatment than the home group in the intention to treat analysis. Predictors with increased risk of treatment failure at any time were age 3-11 months, receiving antibiotics within $48 \mathrm{~h}$ prior to enrolment and use of high polluting fuel. Death rates at 7 or 14 days did not differ significantly. (Difference $-0.0 \% ; 95 \% \mathrm{Cl}-0.5$ to 0.5). The median total treatment cost was Rs. 399 for the home group versus Rs. 602 for the hospital group $(p<0.001)$, for the same effect of $5 \%$ failure rate at the end of 7 days of treatment in the random subsample.

(Continued on next page)
\end{abstract}

\footnotetext{
* Correspondence: drakashbang@gmail.com

${ }^{2}$ Mahatma Gandhi Institute of Medical Sciences, Sewagram, Maharashtra, India

Full list of author information is available at the end of the article
} 
(Continued from previous page)

Conclusions: Home based oral amoxicillin treatment was equivalent to hospital treatment for first $48 \mathrm{~h}$ in selected children of chest indrawing pneumonia and was cheaper. Consistent with the recent WHO simplified guidelines, management with home based oral amoxicillin for select children with only fast breathing and chest-indrawing can be a cost effective intervention.

Trial Registration: ClinicalTrials.gov NCT01386840, registered 25 $5^{\text {th }}$ June 2011 and the Indian Council of Medical Research REFCTRI/2010/000629.

Keywords: Severe pneumonia, Lower chest indrawing, Hospitalization, Oral amoxicillin, Cost effective, Randomized trial

\section{Background}

Pneumonia is the single largest killer of children under the age of five worldwide [1]. The disease kills over two million children under the age of five every year - nearly one fourth $(400,000)$ of these deaths occur in India alone [2]. About half of pneumonia cases in India are caused by bacteria and could be treated with antibiotics. However, only $13 \%$ of Indian children under the age of five with suspected pneumonia receive antibiotics [3].

The 2008 WHO guidelines for treatment of nonsevere pneumonia (cough, fever and fast breathing) recommend health workers to provide oral antibiotics for three days at home but urgent referral for hospitalization for parenteral (injectable) antibiotics and other supportive therapy after administration of first dose of antibiotics, if the child has severe pneumonia (cough, fever, fast breathing and lower chest indrawing) or very severe disease (pneumonia with the presence of WHO defined danger signs) [4]. Often inability to access a referral facility deprives these children from getting appropriate care. For many families, seeking treatment for their children at a health care facility is often logistically and financially burdensome thus denying them early administration of antibiotics within $48 \mathrm{~h}$ that can potentially improve their outcomes. Additionally transport to a distant facility can entail serious delays in effective treatment. Many children with severe pneumonia referred for admission to a hospital could die in transit or reach too sick to be saved [5]. In addition, when hospitalized, the children with severe pneumonia are vulnerable to nosocomial infections in crowded hospital wards and are also at risk of needle-borne infections due to parenteral therapy. Two important studies have addressed such barriers to the recommended treatment of severe pneumonia. The first study was intended to determine whether oral antibiotics are equivalent to injectable antibiotics when both are given in the hospital. This was an open label equivalency study called APPIS (Amoxicillin Penicillin Pneumonia International Study), which was a large multicentre randomized controlled trial comparing injectable penicillin versus oral amoxicillin given for 7 days to children in the hospital [6]. The second study was called "NOSHOTS" (New Outpatient Short-Course Home Oral Therapy for Severe Pneumonia Study) and was a randomized, open-label equivalency trial done at seven study sites in Pakistan and compared initial hospitalization and parenteral ampicillin for $48 \mathrm{~h}$ followed by 3 days of oral amoxicillin at home, to 5 days of home-based treatment with oral amoxicillin [7]. NO-SHOTS showed that home treatment with high-dose oral amoxicillin is equivalent to hospital based treatment with parenteral ampicillin in selected children aged 3-59 months with WHO defined severe pneumonia [7]. Later, another study- the MASS study (Multicenter Amoxicillin Severe pneumonia Study) showed that clinical treatment failure and adverse event rates among children with severe pneumonia treated at home with oral amoxicillin did not substantially differ across geographic areas (Bangladesh, Ghana, Vietnam and Egypt) and hence home-based therapy of severe pneumonia could possibly be applied to a wide variety of settings [8]. Thus oral amoxicillin at home has proven clinically efficacious in various settings across the world for treatment of selected children with WHO defined severe pneumonia. The Lancet Series on Childhood Pneumonia and Diarrhoea has reported that case management is one of the three most effective interventions to reduce pneumonia deaths in children but also noted that the cost effectiveness of these interventions in national health systems needs urgent assessment [9]. So the cost savings or costeffectiveness of home-based oral antibiotic treatment for WHO defined severe pneumonia in childhood would be important to inform public policy and has not been previously evaluated.

Therefore our objective was to assess the efficacy and cost-effectiveness of a 7-day home-based course of oral amoxicillin as compared to oral amoxicillin administered for the first $48 \mathrm{~h}$ in the hospital followed by 5 days of home-administration. 


\section{Methods}

We conducted an open labelled multi-center prospective two-arm randomized clinical trial at 6 referral hospitals in India (Chandigarh, Chennai, Nagpur, Pune, Sewagram and Aligarh) to evaluate the difference of rates of treatment failures of a 7-day course of oral amoxicillin when administered at home as compared to a 7-day course of oral amoxicillin administered for the first $48 \mathrm{~h}$ in the hospital followed by 5 days of home-administration to treat WHO defined severe pneumonia in children aged 3-59 months. In addition to the clinical outcomes, the costs of treating severe pneumonia, the differences in costs of treatment in the two study groups and the costeffectiveness of the two alternative treatment strategy was also assessed in this trial.

The study was approved by the institutional ethics committees of: Indira Gandhi Government Medical College, Nagpur; Post Graduate Institute of Medical Sciences, Chandigarh; Government General Hospital, Chennai; B.J. Medical College, Pune; Mahatma Gandhi Institute of Medical Sciences, Sevagram, Wardha; Jawaharlal Nehru Medical College, Aligarh; the Research Ethics Review Committee, World Health Organization; and the INCLEN Institutional Review Board through the India Clinical Epidemiology Network (IndiaCLEN, dated $18^{\text {th }}$ Nov 2006).

\section{Eligibility}

Children aged 3-59 months with cough/difficulty in breathing of less than 2 weeks duration, lower chest indrawing (LCI), unresponsive to nebulisation, who did not have any of the exclusion criteria (Table 1) and whose parents gave a written informed consent for their participation were enrolled in the study by trained research staff. All included children were administered the first dose of amoxicillin, sent for chest radiology and then reassessed after radiology. Children were randomized to either treatment arms if there was no clinical deterioration or radiographic signs of consolidation, effusion or pneumothorax (using the the WHO manual for standardization of interpretation of chest radiographs for the diagnosis of pneumonia in children) [10].

\section{Randomization}

Random numbers were computer generated, by using variable length permuted blocks at the coordinating site using STATA 10 program. A separate list was generated for each site and the individual patient assignments were placed in a series of sealed opaque envelopes that were opened for serially eligible patients. The eligible study participants were randomly allocated to either the hospital group in which syrup amoxicillin $(50 \mathrm{mg} / \mathrm{kg} / \mathrm{d}$ in two divided doses) was administered in hospital for initial two days by hospital staff followed by administration
Table 1 Exclusion Criteria

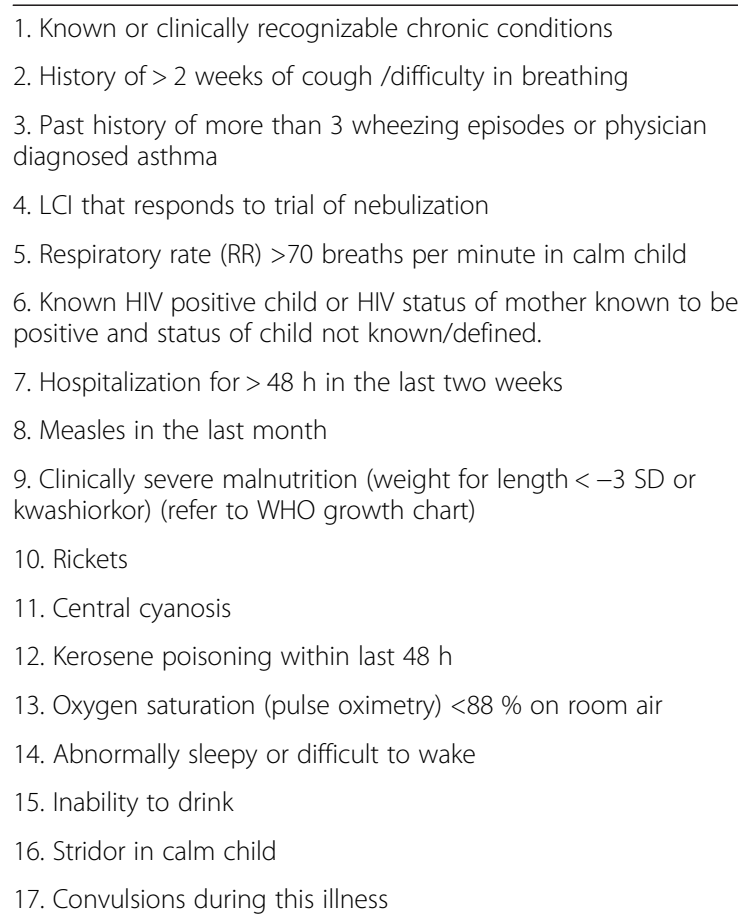

18. Known any antibiotic therapy for $48 \mathrm{~h}$ or more immediately prior to admission

19. Other diseases requiring antibiotic therapy, e.g. Meningitis, tuberculosis, dysentery, etc.

20. Persistent vomiting ( $>3$ episodes of vomiting within $1 \mathrm{~h}$ )

21. Grunting

22. Known prior anaphylactic reaction to penicillin or amoxycillin

23. Severe dehydration according to $\mathrm{WHO}$ guidelines

24. Severe pallor

25. Suspected surgical pathology

26. Living out of the follow-up area of the study (30 kms)

27. Subject previously included in the same trial or already included in another ongoing trial anywhere

28. Presence of radiological consolidation / effusion / pneumothorax

at home for five days by the care-giver, or, to the home group in which the first dose of amoxicillin was administered in hospital and subsequent doses were administered by the care-giver at home for seven days.

\section{Data collection}

Clinical and demographic data was collected at baseline along with throat swab and nasopharyngeal aspirate. Both groups were followed up through home visits on day 3, 5, 8 \& 14 and home group had an additional home visit at $24 \mathrm{~h}$. During these follow-up home visits, data regarding outcomes were collected. This included clinical deterioration of disease any time after enrolment, change of antibiotics, hospitalization, serious adverse 
events considered related to amoxicillin, left against medical advice (LAMA), voluntary withdrawal of consent, or loss to follow up.

\section{Clinical outcomes}

Treatment failure was defined as presence of any one of the following conditions - clinical deterioration of disease any time after enrolment that required change of antibiotics, hospitalization (any time for the children in the home managed group or clinical decision to extend the hospitalization longer than $48 \mathrm{~h}$ in the hospitalized group), an occurrence of a serious adverse event related to amoxicillin, left against medical advice (LAMA), voluntary withdrawal of consent from the study, or loss to follow up. Clinical deterioration was defined as appearance of signs of very severe disease such as persistent vomiting (vomiting repeated three times within an hour due to any reason), central cyanosis, grunt, stridor, abnormal sleepiness or difficulty to wake, inability to drink, $\mathrm{SpO} 2<85 \%$, convulsions, or death [6]. Antibiotics would be changed if there was clinical deterioration, developing a co-morbid condition, or, persisting fever > $98.6^{\circ} \mathrm{F}$ with lower chest indrawing even after $3^{\text {rd }}$ day, or, fever alone at day 5, or, lower chest indrawing alone (non responsive to three doses of nebulisation with bronchodilator) at day 5 (as reported by the mother), or, persistence of fast breathing after day 7 which is unresponsive to three doses of nebulization with bronchodilator. Rigorous training and retraining of the research physicians using standard operating procedures was used to minimize the biases that may arise due to lack of uniformity in assessing clinical signs between treatment groups and across sites. Strong quality monitoring processes were also established. An additional file describes the relevant standard operating procedures in details [see Additional file 1].

\section{Cost outcomes}

Cost data were collected for $17.2 \%$ patients starting from before enrolment till day 14 or till the patient recovered whichever was earlier. Three distinct types of forms were used at enrolment, daily in the hospital and at each visit respectively. The cost data were also collected for those patients who left against medical advice. The forms included information about the service provider and the type of service. We disregarded fixed costs that were common for the two strategies. The protocol driven costs, such as investigations required for the study but not otherwise conducted routinely, were excluded from calculation of these costs. The variable costs i.e. direct medical, direct non-medical and indirect costs of the two treatment arms were measured using micro-costing technique [11].
Direct medical costs included costs of medical resources utilized by the patient at the out-patient and during hospital stay as calculated from the patient's perspective eg cost of medications, physician and nurses services and other paramedical services, bed cost, and the laboratory investigations.

Direct non-medical costs included the cost of travelling to the hospital for the patient and the family, cost of food to the family and patient during hospitalization and other incidental cost to the family attributed to the illness.

Indirect costs were measured by the lost wages for employed parents or guardians attending to the participant.

The median differences in costs and the predictors of total cost were analyzed as cost data was not normally distributed. The incremental cost-effectiveness of the two treatment strategies was also assessed.

\section{Sample size}

Sample size estimates were based to detect equivalence and on the hypothesis that children who were treated with oral amoxicillin at home would experience a failure rate of $15 \%$, and, would be within $5 \%$ of those treated for first $48 \mathrm{~h}$ in hospital. The estimated sample size was 1,234 i.e. 617 per group. The sample size was calculated for the clinical trial but provided $90 \%$ power for a twotailed alternative hypothesis to calculate a mean difference in costs between the two interventions.

\section{Statistical analysis}

Baseline characteristics of the two treatment groups were compared using chi-square tests for categorical variables and ANOVA for continuous variables. We conducted the analysis using both intention to treat (analyzed as randomized) and per protocol analysis (included all clinical causes of treatment failure, but excluded treatment failure due to lost to follow up, LAMA, and voluntary withdrawal from the study). Cox proportional hazards models were used to estimate the relative hazards $(\mathrm{RH})$ of treatment failure in the two groups up to 14 days and to explore associations between the same baseline explanatory covariates (age, feeding status, immunization, antibiotics prior to $48 \mathrm{~h}$, weight for age $\mathrm{Z}$ scores, body temperature, respiratory rates, oxygen saturation, auscultatory wheeze, crackles, radiological infiltrates, number of rooms in the house and type of fuel used for cooking) and outcome. We used forward step wise method and identified explanatory candidate variables $(p \leq 0.1)$ for inclusion in adjusted models as plausible predictors of treatment failure. The Kaplan-Meier curves for the cumulative probability of treatment success were also plotted for the two groups and the overall difference in their rates of treatment success was 
examined using the log-rank test. Statistical analysis was conducted using STATA 10 data analysis software.

\section{Economic analysis}

The medians of the direct medical, direct non-medical and indirect costs and their inter-quartile ranges were calculated. Group differences in median costs of the treatment strategies were assessed using the median test. Univariate analysis was conducted for the predictors of cost variation, such as data on patient demographic characteristics, clinical history, length of stay and other utilization of resources for treatment of this episode of pneumonia before entry of patients into the trial. Multivariable regression analysis (OLS, with log transformation) was also used to predict total costs across the cost categories using pre-randomization variables, the alternate treatment strategies and other covariates that relate to resource consumption. Differences were considered statistically significant if they had a two-tailed $p$ value less than 0.05. The hypothesis, that home treatment is more cost-effective than hospital treatment, was also tested by comparing the cost-effectiveness ratios. The incremental cost-effectiveness was estimated as the difference in the predicted total costs in the numerator and the difference in effects i.e. the number of patients cured (1-treatment failure) or the number of cases of treatment failure avoided in the denominator.

\section{Results}

The study was conducted from October 2008 to March 2011. Of the children screened for WHO defined severe pneumonia, 1118 (16.9\%) were enrolled, 554 were assigned to home treatment, and 564 were assigned to hospital treatment, across six sites in India (Fig. 1). The number of children enrolled from different sites were 377 (33.7 \%), 328 (29.3\%), 316 (28.3\%), 50 (4.5\%), 37 (3.3\%), and 10 (0.9\%) from Chandigarh, Chennai, Nagpur, Sewagram, Aligarh, and Pune respectively. The

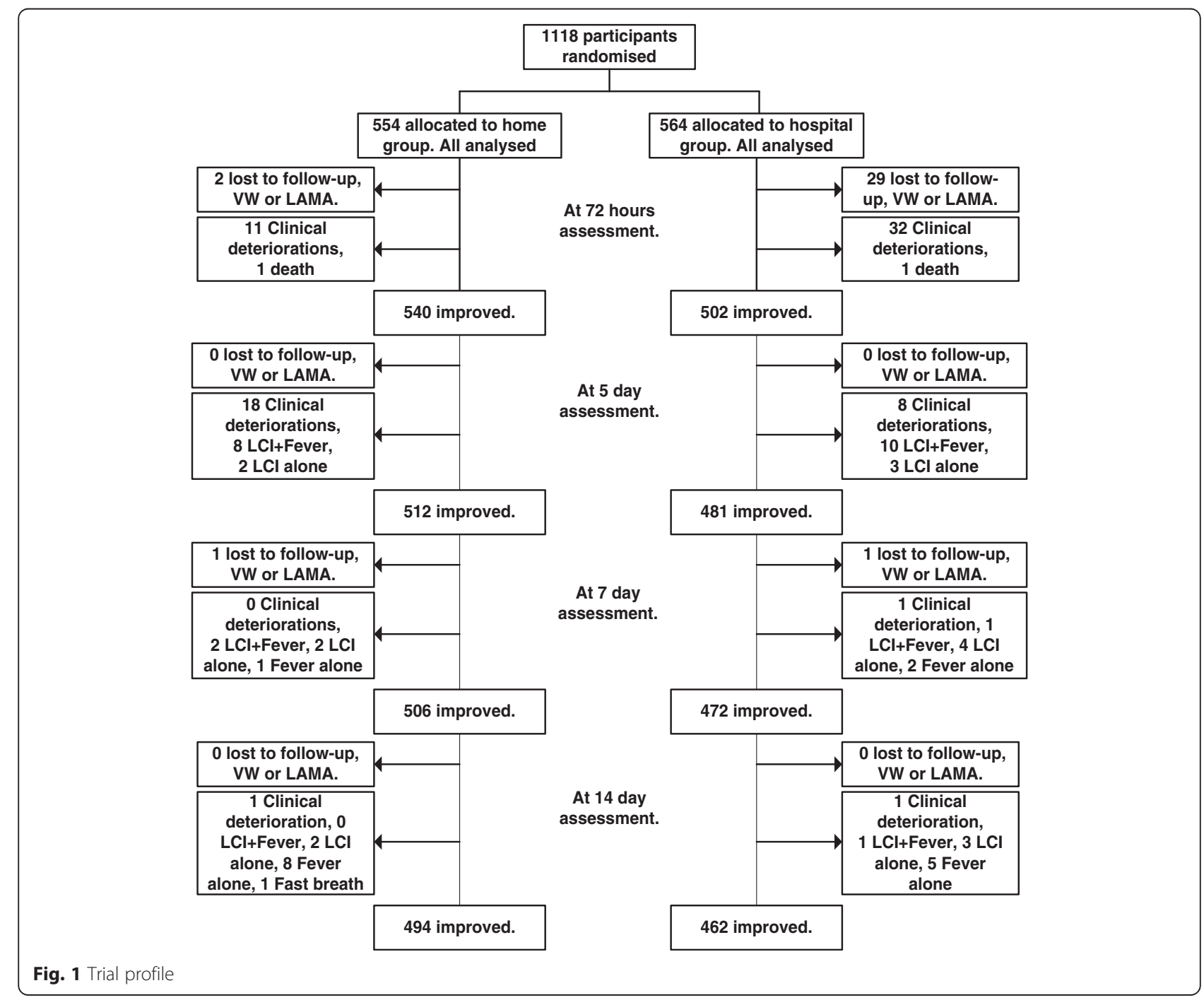


reasons for excluding $83.1 \%$ of screened children are shown in Table 2. The two intervention groups were not statistically different in their baseline characteristics (Table 3).

\section{Clinical outcomes}

The cumulative overall treatment failure (home + hospital) on oral amoxicillin at different time points were $6.8 \%$ at $<72 \mathrm{~h}, 11.2 \%$ at $<5$ days, $12.5 \%$ at $<7$ days, and $14.5 \%$ at $<14$ days by intention to treat and $4 \%$ at $<72 \mathrm{~h}, 8.4 \%$ at $<5$ days, $9.6 \%$ at $<7$ days, and $11.5 \%$ at $<14$ days respectively by per protocol analysis.
The treatment failure rate at 14 days in hospital group was $18.1 \%(102 / 564)$ as compared to $10.8 \%(60 / 554)$ in the home group. There were $30(5.4 \%)$ failures due to clinical deterioration (presence of any one of these conditions - persistent vomiting, central cyanosis, grunt, stridor, abnormally sleepy or difficult to wake, inability to drink, or convulsions) in the home group and 42 (7.4 \%) in the hospital group (Fig. 1) which was not significantly different. The failures due to LAMA or voluntary withdrawal were significantly more in hospital group as compared the home group. [5.3 \% (30/564) vs $0.5 \%(3 / 554) ; p<0.001]$ Kaplan Meier curves for differences between treatment successes in the home

Table 2 Study Screening and Reasons for Exclusion

\begin{tabular}{|c|c|c|}
\hline & Total $^{\#}$ & \\
\hline & $\bar{n}$ & $\%^{\mathrm{a}}$ \\
\hline Screened & 6,634 & \\
\hline Enrolled (\%) & 1,118 & 16.9 \\
\hline Inclusion criteria not satisfied & & \\
\hline Not satisfying Age 3-59 months & 462 & 5.1 \\
\hline Had no lower chest indrawing on examination & 1489 & 16.3 \\
\hline Caretaker is not willing to sign informed consent form & 4060 & 44.5 \\
\hline Exclusion criteria, number (\%) & & \\
\hline Known or clinically recognizable chronic conditions & 224 & 2.5 \\
\hline History of $>2$ weeks of cough / difficulty in breathing & 195 & 2.1 \\
\hline Past history of more than 3 wheezing episodes or physician diagnosed asthma & 267 & 2.9 \\
\hline $\mathrm{LCl}$ that responds to trial of nebulization & 743 & 8.1 \\
\hline Known HIV positive child. HIV status of mother known to be positive \& of child not known/defined & 16 & 0.2 \\
\hline Hospitalization for $>48 \mathrm{~h}$ in the last two weeks & 127 & 1.4 \\
\hline Measles in the last month & 64 & 0.7 \\
\hline Clinically severe malnutrition (weight for length $<-3$ SD or kwashiorkar) & 163 & 1.8 \\
\hline Rickets & 21 & 0.2 \\
\hline Kerosene poisoning within last $48 \mathrm{~h}$. & 17 & 0.2 \\
\hline Antibiotic therapy for $48 \mathrm{~h}$ or more immediately prior to admission & 431 & 4.7 \\
\hline Other diseases requiring antibiotic therapy, e.g. Meningitis, tuberculosis, dysentery, etc. & 28 & 0.3 \\
\hline Known prior anaphylactic reaction to penicillin or amoxycillin & 8 & 0.1 \\
\hline Severe dehydration according to $\mathrm{WHO}$ guidelines & 12 & 0.1 \\
\hline Severe pallor & 19 & 0.2 \\
\hline Suspected surgical pathology & 12 & 0.1 \\
\hline Living out of the follow-up area of the study (30 kms) & 308 & 3.4 \\
\hline Subject previously included in the same trial or already included in another ongoing trials anywhere. & 44 & 0.5 \\
\hline Presence of danger sign ${ }^{c}$ before radiology evaluation & 358 & 3.9 \\
\hline Presence of danger sign after radiology evaluation & 9 & 0.1 \\
\hline Presence of radiological consolidation/ effusion / pneumothorax & 45 & 0.5 \\
\hline Total reasons for exclusion ${ }^{b}$ & 9122 & \\
\hline
\end{tabular}

${ }^{\mathrm{a}}$ Proportion for the presence of exclusion criteria. The denominator is total reasons for exclusion $2 \mathrm{~s}$

${ }^{\mathrm{b}} \mathrm{A}$ child could have more than one reason for exclusions

'Danger signs are presence of any one clinical condition - abnormally sleepy or difficult to wake, persistent vomiting, inability to drink, grunting, stridor, central cyanosis, convulsions, RR $>70$ breaths per minute, $\mathrm{Sp} 02<88 \%$ on room air 
Table 3 Baseline Characteristics in Home and Hospitalized Children

\begin{tabular}{|c|c|c|c|c|}
\hline \multirow[t]{2}{*}{ Baseline characteristic } & \multicolumn{2}{|c|}{ Home $(N=554)$} & \multicolumn{2}{|c|}{ Hospital $(N=564)$} \\
\hline & $n$ & $\%$ & $\bar{n}$ & $\%$ \\
\hline Sex (Female) & 207 & 37.4 & 204 & 36.2 \\
\hline Infants (3-11 months old) & 237 & 42.8 & 277 & 49.1 \\
\hline Children (12-23 months old) & 164 & 29.6 & 138 & 24.5 \\
\hline Children (24-59 months old) & 153 & 27.6 & 149 & 26.4 \\
\hline \multicolumn{5}{|l|}{ Breast feeding indicators(3-59months) } \\
\hline Exclusive breast feeding & 361 & 65.2 & 373 & 66.1 \\
\hline Bottle feeding & 231 & 41.7 & 209 & 37.1 \\
\hline Timely complementary feeding & 431 & 77.8 & 443 & 78.6 \\
\hline Immunization status up-to-date & 516 & 93.1 & 510 & 90.4 \\
\hline Report of antibiotics in $<48 \mathrm{~h}$ prior to enrollment & 56 & 10.1 & 59 & 10.5 \\
\hline Weight-for-age Z score (mean \pm sd) & 554 & $-1.5 \pm 1.3$ & 564 & $-1.6 \pm 1.3$ \\
\hline Weight-for-age Z score $(<-2)$ & 197 & 35.6 & 205 & 36.4 \\
\hline Length/ height $(\mathrm{cm})($ mean $\pm \mathrm{sd})$ & 548 & $75.9 \pm 11.7$ & 560 & $74.7 \pm 12.1$ \\
\hline Temperature $\left({ }^{\circ} \mathrm{F}\right)($ mean $\pm \mathrm{sd})$ & 554 & $99.5 \pm 1.5$ & 564 & $99.6 \pm 1.5$ \\
\hline \multicolumn{5}{|l|}{ Respiratory rate per min (mean $\pm s d$ ) } \\
\hline Infants & 237 & $47.3 \pm 8.9$ & 277 & $48.9 \pm 8.7$ \\
\hline Children & 317 & $43.0 \pm 9.1$ & 287 & $43.9 \pm 9.8$ \\
\hline \multicolumn{5}{|l|}{ Auscultatory wheeze } \\
\hline Infants & $140 / 237$ & 59.1 & $176 / 277$ & 63.5 \\
\hline Children & $155 / 317$ & 48.9 & $121 / 287$ & 42.2 \\
\hline \multicolumn{5}{|l|}{ Crackles } \\
\hline Infants & $174 / 237$ & 73.4 & $211 / 277$ & 76.2 \\
\hline Children & $243 / 317$ & 76.7 & $228 / 287$ & 79.4 \\
\hline \multicolumn{5}{|l|}{ Pulse oximetry (mean $\pm \mathrm{sd}$ ) } \\
\hline Infants & 236 & $96.1 \pm 2.9$ & 277 & $95.9 \pm 3.2$ \\
\hline Children & 317 & $95.9 \pm 3.0$ & 286 & $95.8 \pm 3.1$ \\
\hline Any Infiltrates in chest x-ray & $322 / 530$ & 60.8 & $365 / 540$ & 67.6 \\
\hline No. of rooms in house (mean $\pm s d)$ & 554 & $2.4 \pm 1.4$ & 564 & $2.3 \pm 1.4$ \\
\hline \multicolumn{5}{|l|}{ Fuel used for cooking } \\
\hline Low polluting fuel & $220 / 553$ & 39.8 & $249 / 556$ & 44.8 \\
\hline High polluting fuel & $333 / 553$ & 60.2 & $307 / 556$ & 55.2 \\
\hline Any smoker who smokes in the house & 121 & 21.8 & 129 & 22.9 \\
\hline
\end{tabular}

and hospital group with log rank tests for the intention to treat (ITT) analysis showed that the hospital group was significantly more likely than home children to fail treatment at any time point. (HR 1.79; $95 \%$ C.I. 1.30, 2.46, p<0.01) (Fig. 2) The per protocol analysis, though tended to show a similar trend (RR 1.32), was statistically non-significant $(p=0.10)$. The Cox Regression model showed that infants (3-11 months) and patients who had antibiotics within $48 \mathrm{~h}$ of enrolment had a higher likelihood of failing treatment at any point from enrolment to 14 days (per protocol and intention to treat analysis). Additionally, belonging to the hospital group and residence in homes with high polluting fuels were significantly associated with treatment failure in ITT, because children in the hospital group were more likely to fail treatment at any time than children in the home group (with LAMA accounting for the majority of these failures) (Table 4).

Two children died within the first $72 \mathrm{~h}$, one in the home group, and the other in the hospital group. There were no other serious adverse events. Neither of the deaths were considered to be related to the study treatment with oral amoxicillin. 


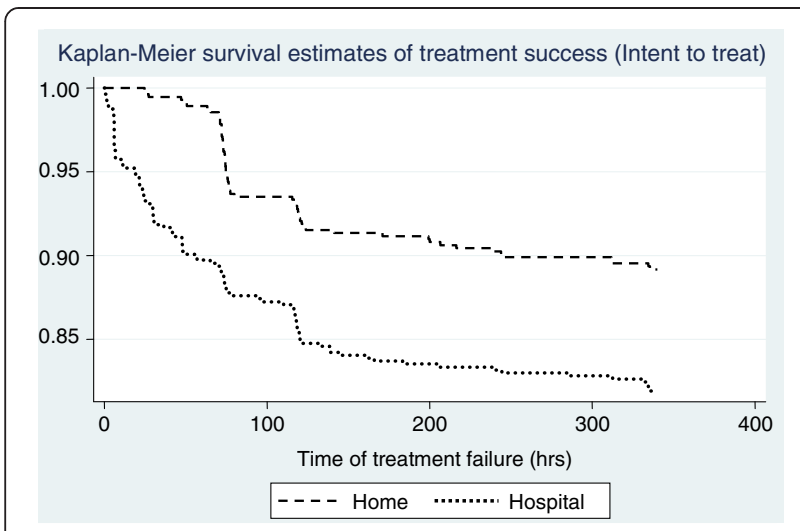

Fig. 2 Kaplan Meier Curves for treatment success rates for intention to treat analysis

\section{Cost outcomes}

The average cost of treating a child at a government hospital in India with subsidized rates was Rs. 567 when patients were hospitalized for only two days. The median total cost for treating at home was significantly less than treating at hospital for the first $48 \mathrm{~h}$. (Rs 399 for home vs. Rs 602 for the hospital group, $p<0.001$ ) (Table 5) The predictors of total mean costs of treatment are shown in Table 6. The patient characteristics associated with higher costs of treatment were age 3-11 months, higher temperature, lower pulse oximetry readings, and presence of auscultatory wheeze. The boot strap cost estimates of Rs.702 (95\% CI 701, 703) for the hospital group and Rs. 427 (95\% CI 427, 428) for the home group were consistent with those determined by the above regression and were significantly higher for the hospital group $(p<0.001)$. Figure 3 shows the plotting of the boot strap estimates $(20,000$ re-samples $)$ on the cost effectiveness plane. It indicates that it is cheaper to be treated at home (all points are below zero in the $\mathrm{Y}$ coordinate of costs) with identical effects (all points are equally distributed on either side of zero in the $\mathrm{X}$ coordinate of effectiveness).

\section{Discussion}

\section{Is oral Amoxicillin effective?}

Our study showed that oral amoxicillin, whether administered at hospital or at home for the first $48 \mathrm{~h}$ was effective in treating WHO defined severe pneumonia in $93.2 \%$ of eligible patients who were otherwise clinically stable and did not have co-morbid conditions. Although the rates of clinical deterioration were similar over the 14 day follow up, the treatment failure rate was more in the hospital group (18.1\% vs $10.8 \%$ ), due to higher rates of LAMA/voluntary withdrawal (5.3\% in hospital group vs $0.5 \%$ in home group), one of the criteria for the

Table 4 Cox Regression Analysis for Treatment Failure Using the Per Protocol and Intention to Treat Analysis up to 14 days

\begin{tabular}{|c|c|c|c|c|c|c|c|c|}
\hline \multirow[t]{2}{*}{ Characteristic } & \multicolumn{4}{|c|}{ Per-protocol analysis } & \multicolumn{4}{|c|}{ Intention to treat analysis } \\
\hline & Hazard ratio & \multicolumn{2}{|c|}{$95 \% \mathrm{Cl}$} & $p$-value & Hazard ratio & \multicolumn{2}{|c|}{$95 \% \mathrm{Cl}$} & $p$-value \\
\hline \multicolumn{9}{|l|}{ Treatment group } \\
\hline Home & 1.00 & & & & 1.00 & & & \\
\hline Hospital & 1.32 & 0.93 & 1.88 & 0.12 & 1.61 & 1.16 & 2.24 & 0.00 \\
\hline \multicolumn{9}{|l|}{ Age group } \\
\hline Infants (3-11 months old) & 1.00 & & & & 1.00 & & & \\
\hline Children (12-59 months old) & 0.65 & 0.45 & 0.93 & 0.02 & 0.69 & 0.49 & 0.96 & 0.03 \\
\hline \multicolumn{9}{|l|}{ Exclusive breast feeding } \\
\hline No & 1.00 & & & & 1.00 & & & \\
\hline Yes & 1.52 & 0.98 & 2.36 & 0.06 & 1.46 & 0.99 & 2.16 & 0.06 \\
\hline \multicolumn{9}{|l|}{ Antibiotics prior to enrollment } \\
\hline No & 1.00 & & & & 1.00 & & & \\
\hline Yes & 1.79 & 1.06 & 3.02 & 0.03 & 1.72 & 1.07 & 2.76 & 0.02 \\
\hline \multicolumn{9}{|l|}{ Fuel used for cooking } \\
\hline Low polluting fuel & 1.00 & & & & 1.00 & & & \\
\hline High polluting fuel & 1.43 & 0.98 & 2.10 & 0.06 & 1.51 & 1.06 & 2.15 & 0.02 \\
\hline \multicolumn{9}{|l|}{ Study Site } \\
\hline Chandigarh & 1.00 & & & & 1.00 & & & \\
\hline Chennai & 0.18 & 0.09 & 0.33 & 0.00 & 0.41 & 0.26 & 0.64 & 0.00 \\
\hline Nagpur & 0.58 & 0.38 & 0.91 & 0.02 & 0.63 & 0.42 & 0.96 & 0.03 \\
\hline Pune & 2.33 & 0.84 & 6.48 & 0.11 & 2.78 & 1.11 & 6.97 & 0.03 \\
\hline
\end{tabular}


Table 5 Treatment costs in different cost categories in the home and hospital group

\begin{tabular}{|c|c|c|c|c|c|}
\hline \multirow[t]{2}{*}{ Type of costs } & \multicolumn{2}{|c|}{ Home } & \multicolumn{2}{|c|}{ Hospital } & \multirow{2}{*}{$\begin{array}{l}P \text { - } \\
\text { value* }\end{array}$} \\
\hline & $n$ & (median $\pm \mathrm{IQR}$ ) & $n$ & (median \pm IQR) & \\
\hline \multicolumn{6}{|l|}{ Cost of outpatient visits } \\
\hline Direct Medical Cost & 94 & $104 \pm 213$ & 97 & $83 \pm 215$ & 0.6 \\
\hline Direct Non Medical cost & 94 & $57.5 \pm 68$ & 97 & $60 \pm 85$ & 0.7 \\
\hline Indirect Cost & 86 & $0 \pm 0$ & 84 & $0 \pm 0$ & 0.5 \\
\hline \multicolumn{6}{|l|}{ Hospital Cost } \\
\hline Direct Medical Cost & 76 & $130 \pm 36$ & 95 & $166 \pm 101$ & $<0.001$ \\
\hline Direct Non Medical cost & 76 & $40 \pm 37$ & 95 & $165 \pm 130$ & $<0.001$ \\
\hline Indirect Cost & 76 & $0 \pm 50$ & 95 & $0 \pm 150$ & 0.02 \\
\hline Total Direct Medical Cost & 94 & $208 \pm 204$ & 97 & $215 \pm 236$ & 0.09 \\
\hline Total Direct Non Medical cost & 94 & $90 \pm 91$ & 97 & $225 \pm 210$ & $<0.001$ \\
\hline Total Indirect Cost & 94 & $0 \pm 100$ & 97 & $0 \pm 150$ & 0.1 \\
\hline Total Cost & 94 & $399 \pm 346$ & 97 & $602 \pm 524$ & $<0.001$ \\
\hline
\end{tabular}

*P-value of median test

composite outcome "treatment failure". This was a conservative estimate of treatment success as there is uncertainty of the clinical outcome and treatment adherence in those who leave the study prematurely. Figure 1 describes frequency of presence of various criteria for treatment failure and of true clinical deterioration. Secondly, children who are hospitalized are closely monitored by skilled research staff for presence of signs of clinical deterioration and are also likely to experience a change in antibiotic (also a criterion for treatment failure) by a treating physician. These could have potentially increased the failure rate in the hospital group. However, clinical deterioration at $<7$ days was not significantly different between the groups indicating that it did not cause a potential bias in this study. Selective randomization of sicker children to the hospital was unlikely as the allocation was concealed. The high rate of LAMA in the hospital group (5.1\%) demonstrates that hospitalization is a barrier to children receiving a full course of treatment and perhaps the caregivers prefer to

Table 6 Predictors of total mean costs for treating WHO defined severe pneumonia

\begin{tabular}{llc}
\hline Variable & $\beta(95 \% \mathrm{Cl})$ & $P$-value \\
\hline Treatment group (Hospital) & $239.8(102.6,377.0)$ & 0.00 \\
Age group (12-59 months old) & $-195.7(-341.6,-49.9)$ & 0.01 \\
Antibiotics prior to enrollment & $-22.7(-297.5,252.1)$ & 0.87 \\
Weight for age Z-score & $-36.4(-94.0,21.2)$ & 0.21 \\
Temperature & $28.1(3.9,52.2)$ & 0.02 \\
Respiratory rate per min & $1.9(-6.1,10.0)$ & 0.64 \\
Pulse oximetry & $-26.9(-50.4,-3.4)$ & 0.03 \\
Auscultatory wheeze & $204.8(59.5,350.1)$ & 0.01 \\
Any infiltrates in chest X-ray & $106.8(-37.7,251.3)$ & 0.15 \\
\hline
\end{tabular}

have their children who otherwise do not have comorbid conditions such as those listed out in the Table 1, treated at home.

The baseline characteristics predictive of treatment failure were known risk factors such as younger age 311 months $[12,13]$, those who received antibiotics less than $48 \mathrm{~h}$ prior to enrollment (perhaps clinically sicker children) and use of solid fuels for household cooking, a known risk factor for poor treatment response [14-18]. Also, all other sites had lower failure rates than Chandigarh. This was mostly because of persistence of LCI at $48 \mathrm{~h}$ contributed mostly by children of hyperreactive airway disease. National Family Health Survey 2005-06 has reported the northern states to have higher prevalence and symptoms of acute respiratory infections [3]. Chandigarh is in the northern states of India and has colder winters than the remaining sites and reports higher incidence of hyperreactive airway disease. Also, Chandigarh site was a referral tertiary care hospital with larger patient load.

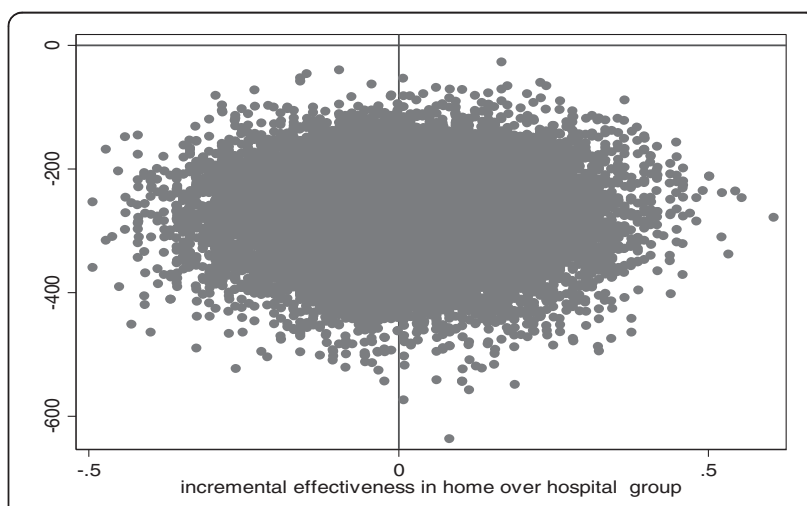

Fig. 320000 Bootstrap re-samples -cost-effectiveness plane 
A recent systematic review has reported results of 4 trials, (2 multi-centric hospital based studies and 2 community based cluster trials) [19]. They reported non inferiority of Oral amoxicillin administered either in hospital or community for treatment of severe pneumonia as compared to standard treatment by injectable penicillin. The two community based cluster trials compared oral amoxicillin administered at home by community health workers to administration of cotrimoxazole in the community followed by standard of care of receiving parenteral therapy at hospital. The hospital based studies compared the use of oral amoxicillin for management of WHO defined severe pneumonia to either injectable penicillin or ampicillin for $48 \mathrm{~h}$. None of these studies compared the use of oral amoxicillin administered for the first $48 \mathrm{~h}$ under hospital supervision to ambulatory management at home with oral amoxicillin. This is the only study that not only reports the overall response of severe pneumonia to oral amoxicillin whether administered at home or hospital but also provides the cost effectiveness of the treatment.

\section{Is oral Amoxicillin cost effective?}

The total cost of treating severe pneumonia in hospital for the first $48 \mathrm{~h}$ was significantly more than being treated at home. There were no significant differences between the two groups in costs of outpatient visits, but significantly higher costs were observed in the hospital group for the costs of hospitalization and for nonmedical costs, which includes expenditure of the caregiver on travel or meal costs when taking care of the child. This was despite the fact that cost of hospitalization was perhaps an underestimate of the true costs as only variable costs were measured, and, because the costs of treatment and hospitalization are subsidized at Indian government hospitals. These results thus suggest that it will be cost efficient to manage children with WHO defined severe pneumonia at home with oral amoxicillin.

This is an important finding because WHO has recommended that children with severe pneumonia must be hospitalised which increases the cost of treatment due to daily bed charge, cost of services of medical personnel, cost of medications, monitoring, lost wages, cost of food for caretakers etc. If the treatment at home for a subgroup of severe pneumonia patients without high risk features, is as effective as treating in the hospital then it will be cost effective to recommend treatment guidelines for management of severe pneumonia at home.

\section{Can these results be generalized?}

Exclusion of children with additional risk factors such as measles, severe malnutrition, and those with radiological consolidation could apparently limit the external validity of the results. However, these were uncommon reasons for exclusion as most seriously ill children with presence of danger signs would be immediately admitted to hospital and would not undergo the screening process. Of the 6634 patients of severe pneumonia that presented to the 6 tertiary care hospitals, only $16.9 \%$ were eligible to participate to receive oral amoxicillin. The most common reason for exclusion in this study at screening was refusal of consent to participate if the child were to be randomized to hospital group. This trend was also observed after the randomization to hospital group when a large number of children left against medical advice and were declared treatment failure by definition despite no clinical deterioration. The other reasons for exclusion were wheeze or lower chest indrawing responding to nebulization (11\%) or having received an antibiotic for longer than $48 \mathrm{~h}$ (4.7\%). Thus, since children with comorbid conditions were not included in the trial these results are largely generalizable to patients with severe pneumonia who don't have co-morbidities or danger signs.

\section{Limitations and strengths}

Excluding patients with consolidation could also exclude those with bacterial pneumonia, while those children with fever, wheeze and infiltrates are more likely to be of nonbacterial causes or viral pneumonias.

Children with allergic bronchitis, asthma, bronchiolitis and viral pneumonia can also manifest with clinical signs of WHO defined severe pneumonia of cough with LCI. Excluding children whose LCI disappeared after nebulization with bronchodilators or those with a past history of wheeze or bronchodilators administration ensured that we minimize inclusion of children with allergic bronchitis or asthma. In developing countries, mixed viral and bacterial infections are not uncommon, and hence need to be treated with antibiotics. However, admitting these children as severe pneumonia will be an additional cost for the Government and for the patient's family.

The limitation in recording the cost data was that it was based partially on recall and partially on documents. It was also difficult to collect the cost data from patients who left against medical advice and those who had treatment failure. The sample size calculation was based on the clinical outcomes and not for the economic analysis. This may have influenced the validity of the cost effectiveness analysis.

Lastly, multiplicity of end points or a composite of end points of treatment failure that includes many conditions such as clinical deterioration, hospitalization, development of co-morbid conditions, changing antibiotics etc. do provide statistical efficiency but at the risk of difficulties with interpretation. Similarly, including treatment failures 
due to leaving against medical advice, lost to follow up or voluntary withdrawal can overestimate treatment failures and may not indicate true clinical deterioration, again causing difficulties with interpretation. Therefore, we analyzed treatment failure on intention to treat as well as per protocol basis.

Finally, this is the only trial that has evaluated the cost effectiveness and efficacy of oral amoxicillin administered at the hospital for first $48 \mathrm{~h}$ as compared to home administered oral amoxicillin to complete a course of 7 day treatment of WHO defined severe pneumonia in children at tertiary care hospitals as it not only reports the overall response of severe pneumonia to oral amoxicillin whether administered at home or hospital but also provides the costs of treatment and the differences in cost in the hospitalized and home group. This is an important strength of the study and will help greatly to guide policy for the case management of severe pneumonia in the developing countries.

\section{Conclusions}

The study results suggest that in selected children aged 359 months with chest indrawing pneumonia and without any of the study exclusion criteria, home based treatment of with oral amoxicillin is equivalent to $48 \mathrm{~h}$ of hospital administered oral amoxicillin followed by home based treatment. However, the results of this study should be generalized with due consideration of the fact that the selected participants had a limited spectrum of presentation which may not be true in the real life scenario.

This study also concludes that cost of treatment of severe pneumonia with oral amoxicillin in the hospital for initial $48 \mathrm{~h}$ followed by continuing it at home for 5 days is significantly more than the cost of treatment with oral amoxicillin at home for 7 days.

Hence, consistent with the recent WHO simplified guidelines, it will be cost effective to manage select stable children with only fast breathing and chestindrawing or WHO defined chest indrawing pneumonia at home with oral amoxicillin.

\section{Additional files}

Additional file 1: Standard operating procedures that were used to train the physicians in assessing clinical signs. (PDF $240 \mathrm{~kb}$ )

Additional file 2: Details of contributorship of the ISPOT study group. (PDF $288 \mathrm{~kb}$ )

\section{Abbreviations}

ANOVA: Analysis of variances; ITT: Intention to treat; LAMA: Left against medical advice; LCl: Lower chest indrawing; OLS: Ordinary Least Squares Regression; Rs: Rupees; WHO: World Health Organization.

\section{Competing interests}

The authors declare that they have no competing interests.

\section{Authors' contributions}

AP conceived the study and prepared the protocol; AP, AB, MS, LD, LRC, AM, SK, and all ISPOT study group members collected the data; LD developed the Manual of Operations and coordinated the staff training; AP did the statistical analysis and prepared the report; $A P$ and $A B$ wrote the manuscript; all the authors- AP, AB, MS, LD, LRC, AM, SK and ISPOT study group members read and approved the final version of the manuscript.

\section{Acknowledgements}

We thank the Data Safety and Monitoring Board members Dr William Macleod (chairperson), Dr Piyush Gupta, Dr Abhaya Indrayan, and Dr Varinder Singh for their oversight and guidance; the Protocol Development Committee members (see details in the Additional file 2) for reviewing the protocol and critically appraising it; Neetu Badhonia, Jitesh Borkar, and Amber Prakash for their help in the statistical analysis; and Dr Ashok K Patwari, Mr John M Pile, and Dr Avinash Ansingkar for reviewing the final report.

This study was funded with grants from MCH STAR, IndiaCLEN, and INCLEN. Amoxicillin was donated by Cipla Pharmaceuticals.

The funding source had no role in any study activities. The publication costs for this paper were funded by the Lata Medical Research Foundation,

Nagpur, India.

The ISPOT Study Group Members: Archana Patel, Leena Dhande, Gopal Agrawal, Girish Charde (Indira Gandhi Government Medical College, Nagpur); Meenu Singh, Sadbhawna Pandit, Pallab Ray, Amit Agarwal (Post Graduate Institute of Medical Sciences, Chandigarh); Luke Ravi Chelliah, C. Ravichandran, Md. Meeran, Saradha Suresh (Institute of Child Health, Chennai); Akash Bang, Manish Jain, Krushna Vilhekar, Deepak Mendiratta, Vijayshree Khairkar (Mahatma Gandhi Institute of Medical Sciences, Sewagram); Ashraf Malik, Uzma Firdaus, Meher Rizvi (Jawaharlal Nehru Medical College, Aligarh); Sandhya Khadse, Chhaya Valvi (B.J. Medical College, Pune).

An additional file describes details of contributorship [See Additional file 2].

\section{Author details}

${ }^{1}$ Lata Medical Research Foundation and Indira Gandhi Government Medical College, Nagpur, India. ${ }^{2}$ Mahatma Gandhi Institute of Medical Sciences, Sewagram, Maharashtra, India. ${ }^{3}$ Post Graduate Institute of Medical Sciences, Chandigarh, India. ${ }^{4}$ Institute of Child Health, Chennai, India. ${ }^{5}$ Jawaharlal Nehru Medical College, Aligarh Muslim University, Aligarh, India. ${ }^{6}$ B.J. Medical College, Pune, India.

Received: 12 December 2014 Accepted: 14 November 2015

Published online: 17 November 2015

\section{References}

1. Walker CL, Rudan I, Liu L, Nair H, Theodoratou E, Bhutta ZA, et al. Global burden of childhood pneumonia and diarrhea. Lancet. 2013;381:1405-16.

2. Rudan I, Boschi-Pinto C, Biloglav Z, Mulholland K, Campbell H. Epidemiology and etiology of childhood pneumonia. Bulletin of the World Health Organization. 2008;86:408-16.

3. International Institute for Population Sciences (IIPS) and ORC Macro. National Family Health Survey (NFHS-3), 2005-06, vol. 1. Mumbai: IIPS; 2007. p. 234-6.

4. World Health Organization: Technical bases for the WHO recommendations on the management of pneumonia in children at first-level health facilities. Geneva: World Health Organization; 1991. Available at: http://www.who.int/maternal_child_adolescent/documents/ ari_91_20/en/. Accessed 17 November 2015.

5. World Health Organization: Home treatment of pneumonia safe and effective, finds study. Available at: http://www.who.int/mediacentre/news/ releases/2008/pr01/en/. Accessed 17 November 2015.

6. Addo-Yobo E, Chisaka N, Hassan M, Hibberd P, Lozano JM, Jeena P, et al. Oral amoxicillin versus injectable penicillin for severe pneumonia in children aged 3 to 59 months: a randomised multicentre equivalency study. Lancet. 2004;364:1141-8.

7. Hazir T, Fox LM, Nisar YB, Fox MP, Ashraf YP, MacLeod WB, et al. Ambulatory short-course high-dose oral amoxicillin for treatment of severe pneumonia in children: a randomised equivalency trial. Lancet. 2008;371:49-56.

8. Addo-Yobo E, Anh DD, El-Sayed HF, Fox LM, Fox MP, MacLeod W, et al. Outpatient treatment of children with severe pneumonia with oral 
amoxicillin in four countries: the MASS study. Trop Med Int Health. 2011;16: 995-1006.

9. Bhutta ZA, Das JK, Walker N, Rizvi A, Campbell H, Rudan I, et al. Interventions to address deaths from childhood pneumonia and diarrhoea equitably: what works and at what cost? Lancet. 2013;381:1417-29.

10. Cherian T, Mulholland EK, Carlin JB, Ostensen H, Amin R, de Campo M, et al. Standardized interpretation of paediatric chest radiographs for the diagnosis of pneumonia in epidemiological studies. Bulletin of the World Health Organization. 2005;83:353-9.

11. Luce BR, Manning WG, Seigel JE, Lipscomb L. Estimating costs in costeffectiveness analysis. In: Gold MR, Siegel JE, Russel LB, Weinstein MC, editors. Cost-Effectiveness in Health and Medicine. New York: Oxford University Press; 1996.

12. Zhang Q, Guo Z, Bai Z, MacDonald NE. A 4 year prospective study to determine risk factors for severe community acquired pneumonia in children in southern China. Pediatr Pulmonol. 2013;48:390-7.

13. Basnet S, Adhikari RK, Gurung CK. Hypoxemia in children with pneumonia and its clinical predictors. Indian J Pediatr. 2006;73:777-81.

14. Jackson S, Mathews KH, Pulanic D, Falconer R, Rudan I, Campbell H, et al Risk factors for severe acute lower respiratory infections in children: a systematic review and meta-analysis. Croat Med J. 2013;54:110-21.

15. Ramesh Bhat $Y$, Manjunath N, Sanjay D, Dhanya $Y$. Association of indoor air pollution with acute lower respiratory tract infections in children under 5 years of age. Paediatr Int Child Health. 2012;32:132-5.

16. Smith KR, McCracken JP, Weber MW, Hubbard A, Jenny A, Thompson LM, et al. Effect of reduction in household air pollution on childhood pneumonia in Guatemala (RESPIRE): a randomised controlled trial. Lancet. 2011:378:1717-26.

17. Mahalanabis D, Gupta S, Paul D, Gupta A, Lahiri M, Khaled MA. Risk factors for pneumonia in infants and young children and the role of solid fuel for cooking: a case-control study. Epidemiol Infect. 2002;129:65-71.

18. Hakim AK, Kharboush IF, Naguib KK, Mortada MM, Noweir KH, El Araby II. Indoor air pollution and acute lower respiratory infections in the first two years of life. J Egypt Public Health Assoc. 1995;70:661-78.

19. Das RR, Singh M. Treatment of severe community-acquired pneumonia with oral amoxicillin in under-five children in developing country: a systematic review. PLoS ONE. 2013;8, e66232.

\section{Submit your next manuscript to BioMed Central and we will help you at every step:}

- We accept pre-submission inquiries

- Our selector tool helps you to find the most relevant journal

- We provide round the clock customer support

- Convenient online submission

- Thorough peer review

- Inclusion in PubMed and all major indexing services

- Maximum visibility for your research 